\title{
Experimental studies on the mode structure of random lasers
}

\author{
Ramy G. S. El-Dardiry, ${ }^{1, *}$ Allard P. Mosk, ${ }^{2}$ Otto L. Muskens, ${ }^{1,3}$ and Ad Lagendijk ${ }^{1}$ \\ ${ }^{1}$ FOM-Institute for Atomic and Molecular Physics AMOLF, Science Park 104, NL-1098 XG Amsterdam, The Netherlands \\ ${ }^{2}$ Complex Photonic Systems, MESA+ Institute for Nanotechnology and Department of Science and Technology, University of Twente, \\ Post Office Box 217, NL-7500 AE Enschede, The Netherlands \\ ${ }^{3}$ School of Physics and Astronomy, University of Southampton, SO17 1BJ Southampton, United Kingdom
}

(Received 17 February 2010; published 22 April 2010)

\begin{abstract}
We investigate the mode structure of a random laser consisting of a porous semiconductor matrix infiltrated with and surrounded by laser dye. The experimental parameters that influence the types of lasing that are observed are identified. The spatial structure of random laser modes is accessed experimentally by a spatially and spectrally selective detection scheme. We show that random laser modes are distinct from laser speckle.
\end{abstract}

DOI: 10.1103/PhysRevA.81.043830

PACS number(s): 42.55.Zz, 42.25.Dd

\section{INTRODUCTION}

The development of nanoscale light sources is of considerable scientific and technological importance. Yet one of the most elementary concepts, the random laser, continues to present many intriguing open questions. In random lasers, optical gain and multiple scattering of light are combined. When the total optical gain overcomes the total losses, lasing occurs [1]. Light that is spontaneously emitted due to vacuum fluctuations inside the multiple scattering medium causes a cascade of stimulated emission events. The first experiments on random lasers showed a clear threshold in the spectral output power as a function of the input power and a narrowing of the emission spectrum above threshold [2-4]. Random laser action has been observed in a great variety of systems including laser crystal powders [2,5], semiconductor scattering media combined with dye molecules [4], and photonic glasses [6]. Recently, significant progress has been made toward realizing random lasing with cold atoms as a gain medium [7].

In important work by Cao et al. [8], spectral spikes were observed on top of a narrowed random laser output spectrum. The origin of these spikes has attracted considerable interest in the literature as it lies at the heart of the complex nature of random lasers. Experimentally, it was shown that narrow spectral features can be induced in both weakly scattering media [9,10], that is, $k \ell \gg 10$, and strongly scattering media $[8,11]$; that is, $k \ell<10$. Here $k$ is the wave number of light and $\ell$ is the transport mean free path. The studies revealed a fundamental difference between systems in which spikes reproduce at a fixed spectral position $[8,11]$ and systems in which the position of spikes is uncorrelated from shot-to-shot [10]. In the case of fixed spectral positions, a mode structure can be associated with a spike [12]. Recent numerical simulations [13] and a two-dimensional analytic theoretical model [14] revealed that random laser modes fluctuate strongly in space. In three previous experimental articles, the spatial extent of these random laser modes was studied $[11,15,16]$.

The observation of modes confined in space has led to the interpretation of random lasing as a manifestation of

\footnotetext{
*dardiry@amolf.nl; http://www.randomlasers.com
}

Anderson localization [8,16]. Anderson localization is an interference effect that brings diffusion of light to a complete halt. The Anderson localization transition, expected to occur around $k \ell_{s} \approx 1$, with $l_{s}$ the scattering mean free path, is hard to reach experimentally in three-dimensional systems. No experiments have been performed that show Anderson localization in both passive and active random media simultaneously. Therefore, an explanation of random lasing in terms of Anderson localization remains elusive. At the same time, the diffusion approach has been able to successfully describe random lasing in the absence of spectral spikes $[17,18]$. An $a b$ initio self-consistent theory based on strong mode interactions that is able to explain spectral spikes in random lasers for weakly scattering media without the need to invoke the concept of Anderson localization has been worked out by Türeci et al. [14,19].

In this article, we experimentally study random lasing phenomena in a porous semiconductor $(\mathrm{GaP})$ infiltrated with and surrounded by laser dye. Using a new experimental arrangement, based on confocal microscopy techniques, we gain access to the microscopic mode structure at the surface of this random material. In Sec. II, the experimental apparatus and the sample preparation are discussed. Measured spatial profiles of random lasing modes are presented in Sec. III A and analyzed in Sec. III B. In Sec. IIIC, the random laser mode structure is compared to speckle in a passive medium. In Sec. III D, several key parameters are identified, such as pump power and pump area, which influence the presence of reproducible spikes in the emission spectrum. Quantitative tools for studying the reproducibility of spectral spikes are given and discussed. We carefully study how laser dye located outside the random medium influences the random lasing emission in Sec. III E.

\section{EXPERIMENTAL METHODS}

In this section, the fabrication of porous GaP samples and the way they are embedded within our optical apparatus are discussed in Sec. II A. The optical experimental setup itself is described in detail in Sec. II B. The experimental method for evaluating the amount of amplification occurring outside the sample is discussed in Sec. II C. 

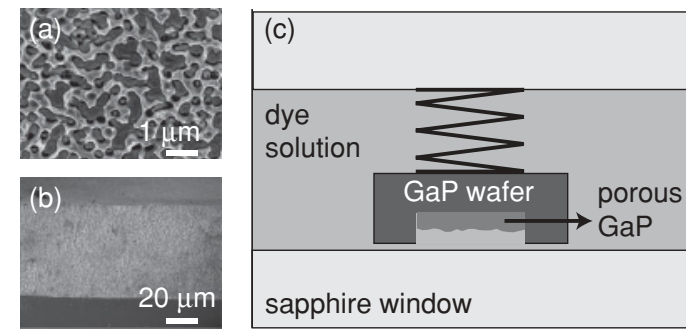

FIG. 1. (a) Top SEM image of a porous GaP sample. (b) A slightly tilted side view of a porous GaP sample. (c) Sample configuration. A light spring pushes the sample onto a sapphire window.

\section{A. Sample fabrication and configuration}

Random laser samples were made by infiltrating strongly scattering porous $\mathrm{GaP}$ with an acidic solution of $20 \mathrm{mM}$ rhodamine 640 perchlorate in methanol (minimal gain length $6 \mu \mathrm{m}$, absorption length $11 \mu \mathrm{m}$, quantum efficiency $>90 \%$ ). The dye solution was put in an ultrasonic bath for $30 \mathrm{~min}$ to ensure the dye was completely dissolved.

The porous GaP samples were fabricated by electrochemically etching a $9 \times 9$-mm piece of a 0.5 -mm-thick crystalline $\mathrm{GaP}$ wafer [MTI corporation, carrier concentration $=2-8 \times$ $10^{17} \mathrm{~cm}^{-3}, n$-type, S-doped, (100) orientation] in a $0.5 \mathrm{M}$ aqueous solution of $\mathrm{H}_{2} \mathrm{SO}_{4}$ under dark conditions. In general, the size of the pores, the pore density, and the shape of the pores depend on the dopant density, the potential at which the sample is etched, and the electrolyte used [20]. A highly reflective top layer is formed during the etching process. To remove this top layer, we used an intermediate etching step at a high potential lying in the regime of passivation [11]. After fabrication, the samples were cleaved and inspected with a scanning electron microscope (SEM). Figures 1(a) and 1(b) show two SEM images of one the porous GaP samples used in our experiments. From a two-dimensional autocorrelation analysis of the top SEM image, we find the typical correlation length at the surface of the sample to be $100 \pm 50 \mathrm{~nm}$.

The transport mean free path of the porous GaP samples in air was determined by an enhanced backscattering experiment $[21,22]$. Infiltration of the porous structure with the dye solution slightly increases the transport mean free path, due to a lower refractive index contrast. After correcting for this lower refractive index contrast [23], we determined the transport mean free path in the two random laser samples used, to be $\ell=0.5 \pm 0.1 \mu \mathrm{m}$ and $\ell=1.4 \pm 0.1 \mu \mathrm{m}$ at $\lambda=632.8 \mathrm{~nm}$. Since the disorder in porous $\mathrm{GaP}$ is quenched (that is, the position of the scatterers does not change in time), the samples are well suited for static speckle and random laser experiments.

For the experiments, the samples were placed in a sample holder which was filled with the $20 \mathrm{mM}$ dye solution [see Fig. 1(c)]. A spring was used to press the porous GaP sample against a 3-mm-thick sapphire window. Both pumping and collection of the random laser emission was done via this sapphire window. The holder was mounted onto two translation stages to allow for selecting different areas on the sample manually.

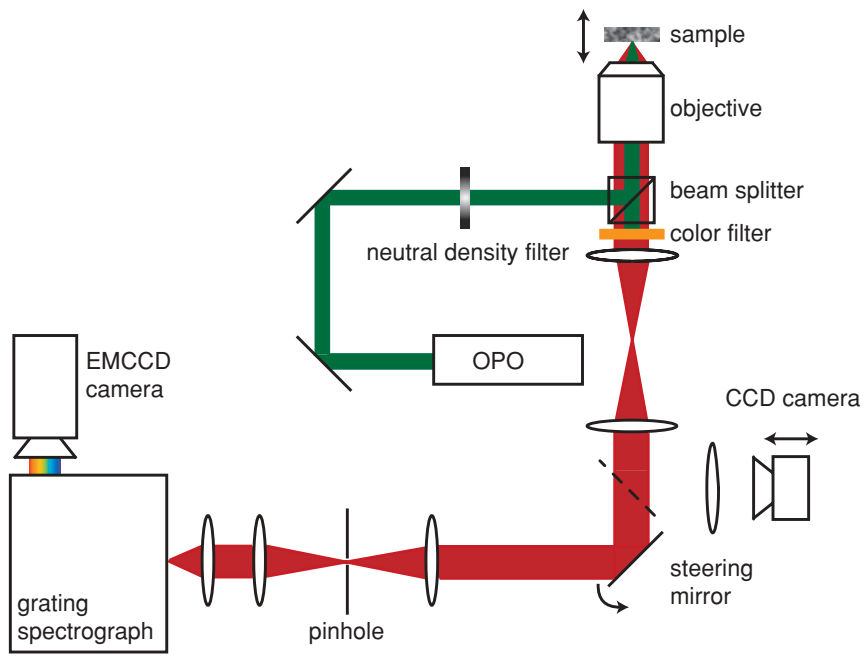

FIG. 2. (Color) Schematic overview of the experimental apparatus. The dashed line represents a flip mirror.

\section{B. Excitation and detection}

Figure 2 shows a schematic overview of the experimental setup. The dye molecules in the random laser sample were excited by 5-ns-long optical pulses, generated by an optical parametric oscillator (Opolette 355-II, Opotek) with a repetition rate of $20 \mathrm{~Hz}$. The wavelength of the pulses was selected in a range between 555 and $565 \mathrm{~nm}$ at an energy below the indirect band gap of $\mathrm{GaP}(548 \mathrm{~nm})$ in order to prevent damage due to absorption. The pump fluence incident on the sample was controlled by means of a tunable reflective neutral density filter. The pump light was focused onto the sample by a long-working-distance microscope objective. The size of the pump spot was controlled by moving the sample out of the focal plane using a translation stage. To determine the size of the diffusive spot profile, the surface of the sample was imaged onto a CCD camera (Mightex). This CCD camera was put on a translation stage to ensure the image was in focus for all positions of the sample. The spatial correspondence between the image on the CCD camera and the actual distances in the sample plane was calibrated using a calibration pattern.

Light emitted by the random laser was collected using the same microscope objective and filtered by a 567-nm-long pass filter (Semrock). To enable two-dimensional spatial mapping of the random laser emission at the sample surface, we introduced a spatially and spectrally selective detection scheme as shown by the red shaded region in Fig. 2. This detection path consisted of a bidirectional steering mirror (FSM-300, Newport), two achromatic lenses ( $f=100 \mathrm{~mm}$ ), and a pinhole $(20 \mu \mathrm{m})$. Two $200-\mathrm{mm}$ relay lenses placed before the steering mirror prevented the beam from walking in the detection path. The scanning experiments were done with a 0.55 NA microscope objective (CFI LU Plan Epi ELWD 50×, Nikon). The spatial resolution was $750 \pm 50 \mathrm{~nm}$. All other experiments were done with a 0.25 NA microscope objective (Leitz). After passing the detection path, the light was detected using a spectrograph (Oriel MS-257, resolution $0.5 \mathrm{~nm}$ ) and an electron-multiplying CCD (EMCCD) camera (C9100-02, Hamamatsu). 


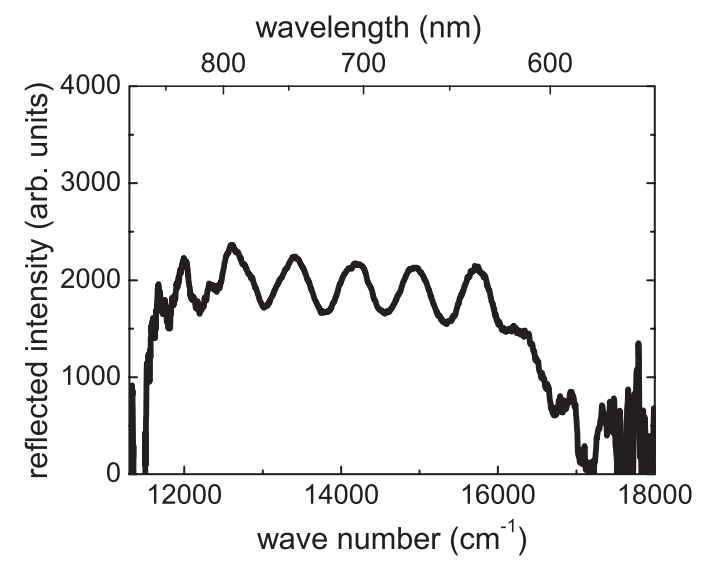

FIG. 3. Interference fringes due to space between the porous $\mathrm{GaP}$ sample and the sapphire window. The sample was illuminated by a white-light supercontinuum source. The shape of the spectrum is corrected for the spectrum of the white-light source.

\section{Determination of sample position}

In the geometry of our experiment, it is unavoidable that a thin fluid layer is present between the multiple scattering sample and the sapphire window. The thickness of this fluid layer is a crucial parameter in our random laser studies since it determines how much amplification of light takes place outside the multiple scattering sample. Typical values for this thickness lie between 5 and $15 \mu \mathrm{m}$. The distance between the sample and the window depends on the particular configuration of the sample inside the sample holder and cannot be controlled manually. In order to determine the thickness of the fluid layer, we used the method of white-light interferometry. Light from a white-light supercontinuum source (Fianium SC-450) was coupled into the setup and subsequently analyzed with a broadband fiber spectrometer (Ocean Optics USB 4000). An example of a reflection spectrum is shown in Fig. 3. In this figure the reflected intensity is shown as a function of wave number. Clear interference fringes are visible. The intensity has been corrected for the spectral shape of the white-light source. The fringes are due to multiple reflections between the sapphire window and the porous $\mathrm{GaP}$ sample. Fringes with higher visibility are observed when crystalline $\mathrm{GaP}$ is illuminated. The spacing of the fringes, the free spectral range, is dependent on the distance between the sample and the window, $L$, by [24] $\Delta v_{\text {fsr }}=c /(2 n L)$. Here $\Delta v_{\text {fsr }}$ is the free spectral range, $c$ is the speed of light in vacuum, and $n$ is the refractive index of the medium enclosed by the two reflecting surfaces.

\section{RESULTS AND ANALYSIS}

In this section we discuss the outcome of three different experiments with porous $\mathrm{GaP}$ random lasers. In general, a power threshold is present around which the emission spectrum changes. For small excitation areas $\left(<1000 \mu \mathrm{m}^{2}\right)$ the emission spectra above threshold become dominated by spikes, whereas for large excitation areas the output spectrum is narrowed but smooth. The different character between the spectra above threshold is striking in Fig. 4 in which

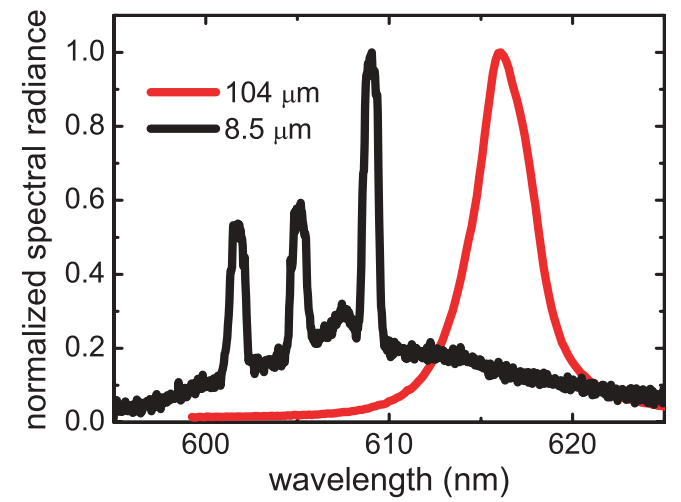

FIG. 4. (Color online) Emission spectra of a porous GaP random laser above threshold for two different excitation conditions. The black line shows the spectrum above threshold for a random laser with a fluorescent profile of $8.5 \mu \mathrm{m}$ in radius. Clear spikes are observed. The red line shows the spectrum above threshold for a random laser with a fluorescent profile of $104 \mu \mathrm{m}$ in radius. The emission spectrum is narrowed, but no spikes are observed.

normalized single-shot spectra 1.3 times above threshold are plotted for a small excitation spot $(8.5 \mu \mathrm{m}$ in radius, $\left.0.078 \mu \mathrm{W} / \mu \mathrm{m}^{2}\right)$ and a large excitation spot (104 $\mu \mathrm{m}$ in radius, $\left.5.2 \times 10^{-3} \mu \mathrm{W} / \mu \mathrm{m}^{2}\right)$. For the small pump spot, clear spikes are visible. For the large pump spot, the output spectrum consists of a smooth narrowed amplified spontaneous emission (ASE) spectrum.

In a first experiment, described in Sec. III A, the spatial structure of random laser modes associated with spectral spikes is measured using confocal microscopy techniques. In Sec. III B, the stability of the spatial mode maps is analyzed. The size of random laser modes is compared to the size of speckles in Sec. IIIC. Two experiments to investigate the presence and reproducibility of spectral spikes under various experimental discussions are presented in Sec. IIID and the results are discussed in Sec. III E.

\section{A. Spatial structure of random laser modes}

We studied the spatial structure of random laser modes in the regime where spikes are clearly visible in the output spectrum. Spatial maps of the entire region excited by the pump spot in the random laser sample were made by confocal scanning (excitation energy: $0.5 \pm 0.1 \mu \mathrm{J}$ per pulse). The $x$ position of the detection area on the sample was scanned in steps of $350 \mathrm{~nm}$, the $y$ position in steps of $420 \mathrm{~nm}$. The total scan comprised $41 \times 41$ pixels. At every pixel in the scan, 25 spectra were measured with an exposure time of $0.2 \mathrm{~s}$. The spectra were smoothed by adding four adjacent data points in order to average out the readout noise of the EMCCD camera. Figure 5 shows spatial intensity maps for spikes at seven different wavelengths together with the $50 \%$ intensity contour of the diffuse fluorescence spot (diffusion of both the pump and the fluorescence light increases the excited volume inside the sample). The profile of the fluorescence spot follows the profile of the population inversion in the gain medium. The diffuse fluorescence spot is slightly elliptical, which allows us to study the effect of this asymmetry on the mode structure. 
(a)

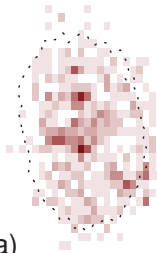

(d)

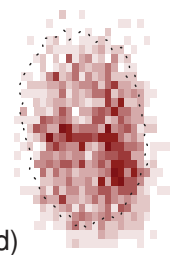

(g)

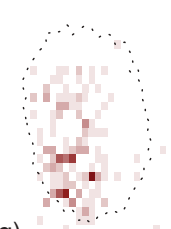

(h)

(e)

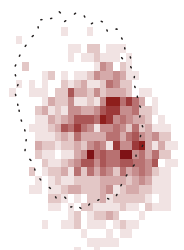

(c)
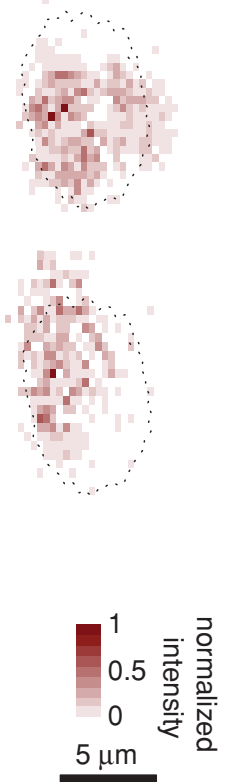

FIG. 5. (Color online) Experimentally determined spatial mode maps for seven modes at seven different wavelengths. (a) $603 \pm$ $0.2 \mathrm{~nm}$, (b) $604.3 \pm 0.2 \mathrm{~nm}$, (c) $607 \pm 0.2 \mathrm{~nm}$, (d) $607.8 \pm 0.2 \mathrm{~nm}$, (e) $609.5 \pm 0.2 \mathrm{~nm}$, (f) $610.8 \pm 0.2 \mathrm{~nm},(\mathrm{~g}) 613.3 \pm 0.2 \mathrm{~nm}$. The black dotted line is the $50 \%$ intensity contour of the diffuse fluorescence spot. The color coding for each plot is independent. In (h) an optical parametric oscillator speckle pattern $(605.1 \mathrm{~nm})$ is shown.

The maps are constructed by adding the spike intensity for the 25 measured spectra at one pixel.

The spatial mapping experiment gives a unique insight in how random lasers work. Figure 5 reveals three key characteristics of modes in GaP random lasers. First, modes of different frequency have a significant spatial overlap at the surface. Second, the intensities of the modes fluctuate in space. Third, the positions of the modes do not always coincide with the peak intensity of the pump spot.

\section{B. Stability analysis of spatial mode maps}

Spatial mode maps can be constructed from the raw data for every wavelength. In this rather technical section, we determine at which wavelengths the spatial mode maps are reproducible in order for us to be able to separate useful information from noise.

The Pearson correlation coefficient between two different data sets, $x$ and $y$, is defined as [25]

$$
P \equiv \frac{\sum_{i}\left(x_{i}-\bar{x}\right)\left(y_{i}-\bar{y}\right)}{\sqrt{\sum_{i}\left(x_{i}-\bar{x}\right)^{2} \sum_{i}\left(y_{i}-\bar{y}\right)^{2}}} .
$$

The correlation coefficient is one when the data sets are fully correlated, and zero when they are not correlated at all. For every wavelength, we now construct two spatial mode maps: the first map is calculated using 12 out of the 25 available spectra per pixel, the second map is calculated using the remaining 13 spectra. The Pearson correlation coefficient between these two maps, $P_{S}$, is calculated for every

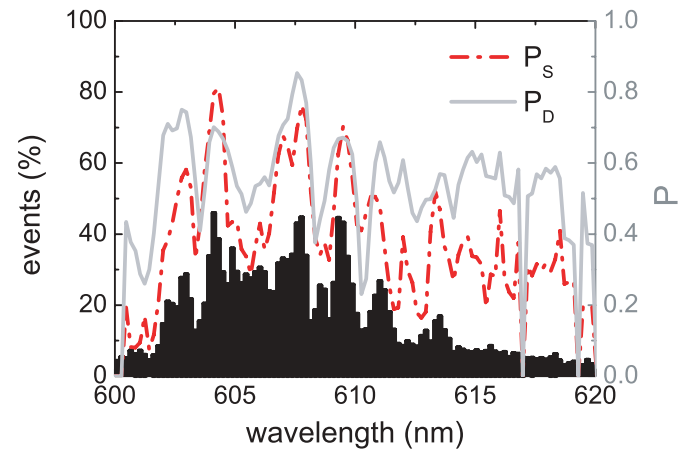

FIG. 6. (Color online) The black histogram denotes the percentage of pixels on which a spike with a certain wavelength is detected (left $y$ axis). The red dashed-dotted line is the correlation coefficient between two subsets $\left(P_{S}\right.$, right $y$ axis) that indicates the stability of the measurement, the gray continuous line is the correlation coefficient between the mode at a certain wavelength and the fluorescence diffusive spot profile ( $P_{D}$, right $y$ axis).

wavelength. In this case, the subscript $i$ in Eq. (1) denotes a particular pixel in the map.

Figure 6 shows $P_{S}$ as a function of wavelength together with a histogram of the percentage of pixels that contain a certain spike. The Pearson correlation coefficient between the random laser modes and the fluorescence spot profile, $P_{D}$, is also calculated from Eq. (1) and shown in Fig. 6. We consider spatial maps at wavelengths where $P_{S}$ is higher than 0.5 to be sufficiently stable for further analysis. Seven modes fulfill this criterion, namely, all the modes shown in Figs. 5(a)-5(g). Furthermore, from the high values of the cross-correlation coefficient we deduce all modes have a significant overlap with the diffuse fluorescence spot profile.

\section{Size of random laser modes and speckle}

The grainy intensity profile of random laser modes might at first glance resemble speckle. To compare the size of random laser modes with the size of speckles, we study the intensityintensity autocorrelation

$$
C(\Delta r) \equiv \frac{\sum_{x, y}[I(x, y)-\langle I(x, y)\rangle]\left[I\left(x^{\prime}, y^{\prime}\right)-\left\langle I\left(x^{\prime}, y^{\prime}\right)\right\rangle\right]}{\sum_{x, y}[I(x, y)-\langle I(x, y)\rangle]^{2}}
$$

Here $I(x, y)$ denotes the measured intensity at pixel $(x, y)$ and $(\Delta r)^{2}=\left(x^{\prime}-x\right)^{2}+\left(y^{\prime}-y\right)^{2}$. The triangular brackets denote ensemble averaging. Figure 7 shows the $2 \mathrm{D}$ ensemble averaged autocorrelation of the seven converged random laser modes and of 20 speckle patterns in reflection obtained by illuminating the sample with light $(605.1 \mathrm{~nm})$ generated by the optical parametric oscillator (OPO). The ensemble average for the random laser modes was constructed by averaging the seven stable mode profiles. The mode profiles were shifted laterally to let their centers of mass overlap. Not performing this centering procedure does not affect the outcome significantly. For the speckle pattern the ensemble average is obtained by averaging over 20 different realizations of disorder. 

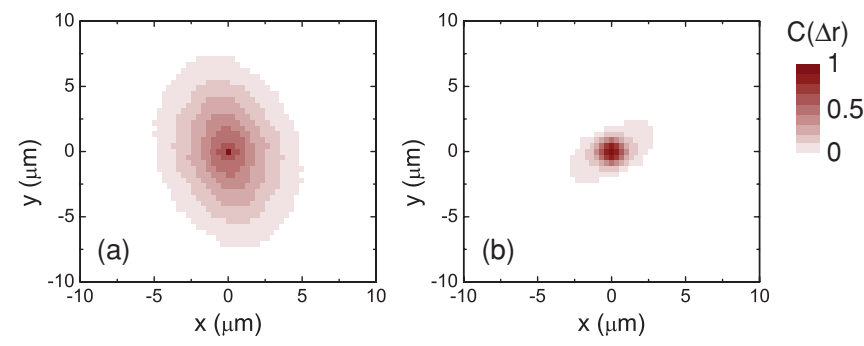

FIG. 7. (Color online) Two-dimensional ensemble averaged normalized autocorrelation plot for (a) random laser modes and (b) speckle.

In Fig. 8, we plot cuts of the two-dimensional data of Fig. 7. The red line is a theoretical prediction from Shapiro for the speckle size inside a random medium [26], given by $\left[\sin k_{0} \Delta r /\left(k_{0} \Delta r\right)\right]^{2} \exp (\Delta r / \ell)$, convolved with the microscope's point spread function. In this theoretical curve we neglect the minor adjustments made by Freund and Eliyahu [27] for correlations on the surface of a random medium. The point spread function was corrected for the presence of the thick sapphire window, which changes the exit pupil and thereby decreases the effective numerical aperture of the microscope objective from 0.55 to 0.3 , as was determined from HeNe speckle measurements (as a side remark, we note that polarization does not influence the normalized autocorrelation of speckle). The first point in the random laser autocorrelation graph accumulated all remaining experimental noise due to the intrinsic fluctuations in intensities of spikes; therefore, the second point in the graph was used to normalize the data. From
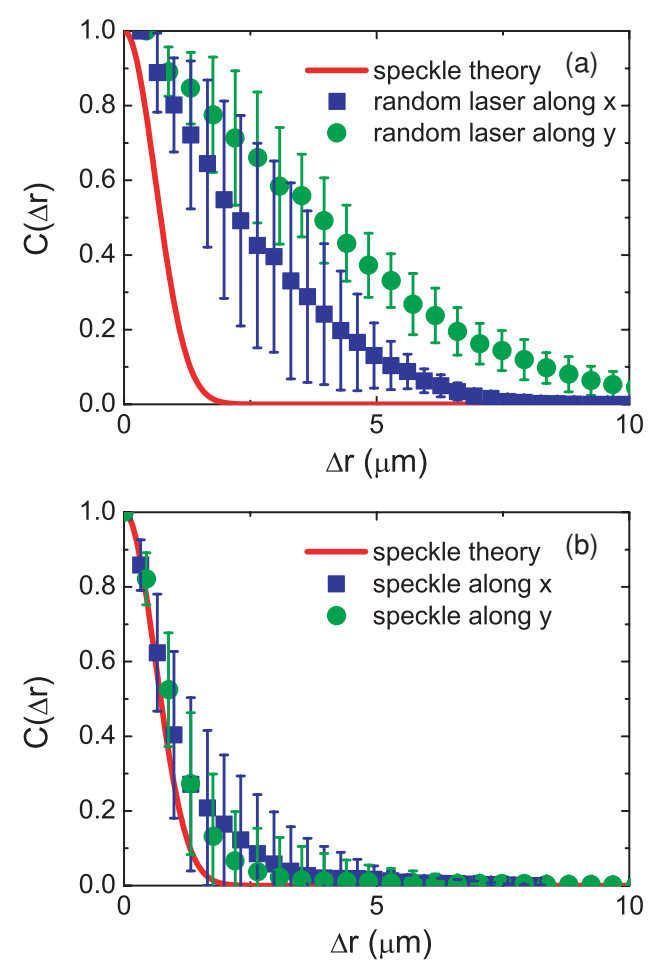

FIG. 8. (Color online) Ensemble averaged intensity-intensity autocorrelation for (a) random laser modes and (b) speckle along different directions. The red solid line is a theoretical prediction for the speckle size. The bars represent the standard deviation. the full width at half maximum values of the autocorrelation graphs we find the size of random laser modes to be $3.0 \pm$ $0.7 \mu \mathrm{m}$ and the size of the speckles to be $0.8 \pm 0.1 \mu \mathrm{m}$. This measured size of the random laser modes is also an order of magnitude larger than the correlation length of the material disorder in porous GaP. The size of the speckles is in good agreement with the theoretically expected value. The asymmetry that was present in the profile of the pump spot also appears in the autocorrelation of the random laser modes. This asymmetry in the autocorrelation indicates that the spatial structure of the measured random laser modes is to some extent influenced by the size of the pumped volume. Furthermore, in contrast to speckle in a passive medium, random laser modes do not span the entire excited area; instead, random laser modes are confined.

\section{Statistics and reproducibility of spectral spikes}

In general, spectral spikes require a careful tailoring of experimental conditions. The intensities of the spikes and in some case the spectral positions differ from shot to shot. Studies on the chaotic behavior of spikes have recently been initiated by Mujumdar et al. [28] and Wu and Cao [29]. To find out more about the nature of the spikes we conducted two additional experiments where no pinhole was present in the setup. In the first experiment, we analyze for the first time the role of the excitation area on spike statistics. Fifty singleshot emission spectra were measured for different excitation areas at a fixed value for the pump fluence, namely $0.07 \pm$ $0.01 \mu \mathrm{W} / \mu \mathrm{m}^{2}$. Second, to understand the role of light amplification outside the random medium, the reproducibility of spectra containing spikes were studied for different distances between the sample and the sapphire window using a similar data analysis as used by Mujumdar et al. [28].

Figure 9 shows the number of spikes per single shot and the relative height of the spikes in the spectrum versus the excitation area. Peaks in the spectra were named a spike when the spike intensity was higher than eight times the standard deviation of the noise and when data points adjacent to the spike within the spectral resolution were lower than the spike value itself. The relative height of spikes increases when the excitation area decreases. However, for all values of the pump spot area we find spikes in the spectrum. Similar experiments

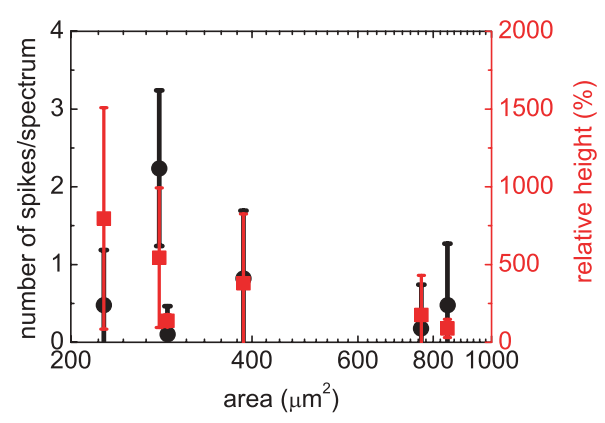

FIG. 9. (Color online) Role of excitation area on statistics of spectral spikes. The number of spikes per spectrum (in black) and the relative height of a spike (in red) are plotted versus the excitation area in a semilog plot. The data points represent the mean of the data, the bars the standard deviation. 


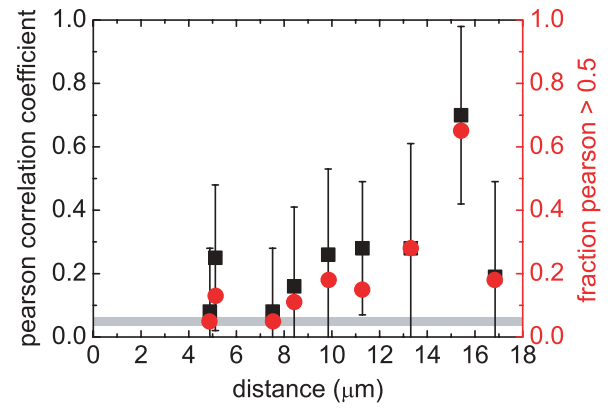

FIG. 10. (Color online) Reproducibility of spike spectral position for different distances between the sample and sapphire window. The black squares represent the mean values of the Pearson correlation coefficient between single-shot spectra; the bars represent the standard deviation. The red circles represent the fraction of coefficients between two single-shot spectra that is higher than 0.5. The gray bar indicates the fraction of coefficients that are higher than 0.5 between single-shot spectra taken at different positions on the sample.

were performed for different spots on the sample and yielded the same trends.

The reproducibility of spikes in spectral position is studied by calculating the Pearson correlation coefficient as defined in Eq. (1) for several distances between the sample and the sapphire window. Now $i$ in Eq. (1) denotes a particular position in the spectrum. The correlation coefficient is 1 when the spectra are fully correlated and 0 when they are not correlated at all. For every distance, 50 single-shot spectra above threshold were recorded, the fluorescence background was subtracted, and the spectra containing spikes were selected for further analysis.

In Fig. 10 the calculated mean of the correlation coefficient is represented by the data points and the standard deviation is used as an error estimate. For all distances between the sample and the sapphire window, the correlation coefficient between the different spectra is small $(<0.5)$ except for the one data point at $15 \mu \mathrm{m}$. It is therefore tempting, but incorrect, to conclude from this analysis that the spikes appear chaotically in the spectrum and reproduce poorly. In fact, spikes do reproduce in spectral position, as can be deduced from a high value $(>0.5)$ of the correlation coefficient of some spectra pairs. Therefore, the fraction of coefficients that is higher than a specified value is a better way to study the reproducibility of spectral spikes. The fraction of coefficients higher than 0.5 is plotted in Fig. 10. The gray bar represents the fraction of correlation coefficients higher than 0.5 between spectra taken at different positions on the sample. Above a distance of $8 \mu \mathrm{m}$ the fraction of highly correlated spectra taken at one position becomes significantly higher than this background value. Hence, we conclude the reproducibility of spikes slightly increases for larger values of the distance between the window and sample.

Although the reproducibility of spikes is low when the liquid layer between the sample and the sapphire window is small, the spectra itself still contain valuable information about the spectral structure of random laser modes. Figure 11 shows the normalized ensemble averaged autocorrelation, $C(\Delta \lambda)=\langle I(\lambda) I(\lambda+\Delta \lambda)\rangle /\left\langle I(\lambda)^{2}\right\rangle$, for two different series of single-shot spectra. The autocorrelation of the spectra

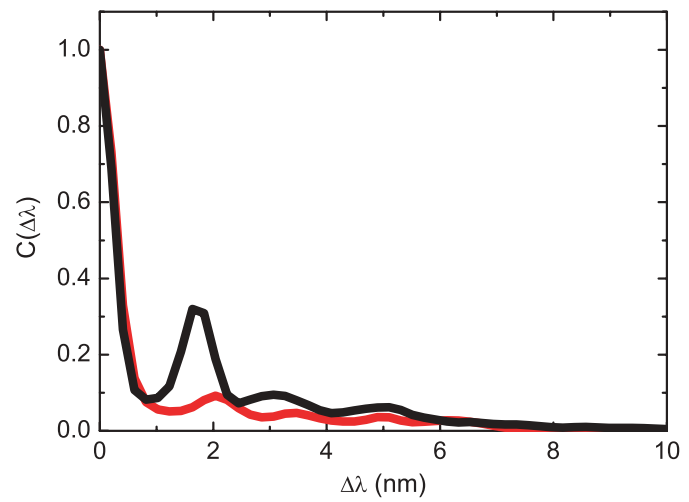

FIG. 11. (Color online) Normalized spectral autocorrelation of single-shot spectra recorded with a distance between sample and sapphire window of $4.9 \mu \mathrm{m}$ (black line) and $15.4 \mu \mathrm{m}$ (red line).

has an oscillatory behavior with a typical oscillation period between 1 and $2 \mathrm{~nm}$. This oscillatory behavior of the spectrum autocorrelation has been observed previously in studies of colloidal dye suspensions and was attributed to spectral hole burning $[29,30]$.

\section{E. Influence of dye surface layer on random laser emission}

The relative height of spectral spikes decreases with excitation area. The spikes, in other words, disappear in the ASE background for larger pump spots. It has been suggested in the original article by Letokhov [31] and more recently by Yamilov et al. [32] that a reduction of the system volume indeed facilitates the observation of spectral spikes due to a decrease in the total number of modes. There is, however, a second, experimental, reason for the decrease of the spike height for larger pump spots that obscures this effect. The small layer of dye solution located between the sample surface and the window can also give rise to ASE. In fact, we observed ASE in the cases of nonetched GaP and weakly scattering samples. The threshold for observing ASE in this thin dye layer decreases for larger excitation areas due to the presence of longer paths that receive more gain. For excitation areas larger than $1000 \mu \mathrm{m}^{2}$ the threshold for ASE in this dye layer is lower than the threshold for observing spikes. Hence, this dye layer prevents studying effects taking place inside the porous $\mathrm{GaP}$ for large excitation spots.

The high concentration of dye molecules in our system causes the gain length in the system to be small $(6 \mu \mathrm{m}$ when all dye molecules are simultaneously excited to the upper laser level). Therefore, spikes in the emission spectrum could already arise due to lasing between two weakly reflecting interfaces. For example, a distance of $16 \mu \mathrm{m}$ between crystalline $\mathrm{GaP}(R=0.2)$ and the aforementioned sapphire window $(R=0.02)$ results in a tiny dye laser under the conditions of our experiment. By comparing the absolute intensity of an unfocused coherent beam from crystalline $\mathrm{GaP}$ and porous $\mathrm{GaP}$, we measure the coherent-beam reflection coefficient of porous $\mathrm{GaP}$ in exact backscattering to be $25 \pm 5 \%$ of the reflection coefficient of crystalline GaP, that is, $R=0.05 \pm$ 0.01 for the porous GaP. The reflection coefficient of porous $\mathrm{GaP}$ is much lower than the reflection coefficient of crystalline 
$\mathrm{GaP}$, because scattering randomizes the reflection direction and the effective refractive index of porous $\mathrm{GaP}$ is lower than the refractive index of crystalline $\mathrm{GaP}$. In the case of porous $\mathrm{GaP}$ a spacing of $21 \mu \mathrm{m}$ between the sample and the window is needed for coherent lasing outside the sample.

Experimentally, we observe spikes for all thicknesses of the fluid layer between the sample and the sapphire window. At the same time, the reproducibility of spectral spikes is slightly enhanced for larger thicknesses of the fluid layer. However, the measured thicknesses for the dye layer are still too short to explain the spikes by standard lasing action caused by the averaged reflectivity of the sample and the reflectivity of the window. Hence, we attribute reproducible spikes to lasing originating partly from within the porous GaP. The alternative possibility, that is, lasing between the window and areas on the sample that have a higher-than-average reflection coefficient, is highly unlikely, since the measured ensemble averaged random laser mode size is an order of magnitude larger than the correlation length of the microscopic disorder in porous $\mathrm{GaP}$. The fact that the spectral mode spacing remains between 1 and $2 \mathrm{~nm}$ for all thicknesses of the fluid layer is another clear indication that the amplification takes part partly within the porous $\mathrm{GaP}$ and is not due to lasing between the sample interface and the sapphire window.

The experiments do show that surface effects play a more important role than was previously assumed. For small distances (in terms of the gain length) between the sample and the sapphire window, most amplification takes place inside the random medium. Due to the high number of modes $\left[(14 \pm 5) \times 10^{3}\right]$ available in such a medium [33], there are many possible laser modes. Therefore, which of the available modes turn "on" depends on statistical fluctuations inside the dye solution and change from shot to shot. In such a situation the spectral position of the spikes cannot be determined a priori.

\section{CONCLUSION AND DISCUSSION}

In the field of random lasing three distinct forms of lasing have been reported in the past: ASE in the form of a narrowing of the emission output spectrum, lasing in the form of a chaotic appearance of spectral spikes, and lasing in the form of reproducible spectral spikes.

We observed these three different types of lasing in and around a porous $\mathrm{GaP}$ sample infiltrated with laser dye. For large excitation volumes (i.e., large compared to the transport mean free path and the gain length), ASE dominates the emission spectrum. This ASE is due to a layer of dye between the sample and the sapphire window. For small pump spots that are comparable to the gain length and the transport mean free path, spectral spikes start dominating over the ASE spectrum. Depending on the distance between the sample and the holder window, the spikes reproduce in spectral position or appear chaotically in the spectrum. The reproducibility of spectral spikes is enhanced for larger distances, indicating that the relatively large extent of modes outside the random medium is a key property of stable random laser modes.

The intense debate in the experimental literature on the origin of spikes $[8,10,11,16]$ can partly be attributed to the fact that in these studies very different systems were taken into consideration. The outcome of our experiments emphasizes that random lasing is predominantly an effect occurring near the boundary of the multiple scattering system [14,19]. The results apply to many random laser experiments, since pumping of random laser systems is almost always done via the outside, and the result is monitored in reflection. The combination of multiple scattering and short pump absorption lengths prevents random laser action from taking place in the bulk of the sample. In such experimental configurations the leakiness of random laser modes to the outside is bound to play an important role.

By studying the spatial intensity-intensity autocorrelation of the random laser modes that are associated with spectral spikes, we are able to compare random laser modes directly with diffusive phenomena for the first time. The structure of these modes on the surface of the sample deviates from speckle. Hence, the gain in an active medium acts as a selection mechanism that chooses a subset of the modes available in the medium to start lasing. The modes are shown to have a significant spatial overlap at the surface, making gain competition effects such as spatial hole burning very plausible.

We hope our work invites experimentalists and theoreticians to compare spectral spikes in random lasers directly with diffusive phenomena and by doing so deepen the understanding of the relation between random lasing, diffusion, and Anderson localization. For example, studying the probability density function of wave functions at the surface of a random laser would be of great interest [34].

\section{ACKNOWLEDGMENTS}

We thank Sanli Faez and Karen van der Molen for stimulating and helpful discussions. Robb Walters is thanked for his careful reading of the manuscript. This work is part of the research program of the "Stichting voor Fundamenteel Onderzoek der Materie (FOM)," which is financially supported by the "Nederlandse Organisatie voor Wetenschappelijk Onderzoek (NWO)."
[1] D. S. Wiersma, Nat. Phys. 4, 359 (2008).

[2] V. M. Markushev, V. F. Zolin, and C. M. Briskina, Zh. Prikl. Spektrosk. 45, 847 (1986).

[3] C. Gouedard, D. Husson, C. Sauteret, F. Auzel, and A. Migus, J. Opt. Soc. Am. B 10, 2358 (1993).

[4] N. M. Lawandy, R. M. Balachandran, A. S. L. Gomes, and E. Sauvain, Nature (London) 368, 436 (1994).
[5] M. A. Noginov, N. E. Noginova, H. J. Caulfield, P. Venkateswarlu, T. Thompson, M. Mahdi, and V. Ostroumov, J. Opt. Soc. Am. B 13, 2024 (1996).

[6] S. Gottardo, R. Sapienza, P. D. García, A. Blanco, D. S. Wiersma, and C. López, Nat. Photonics 2, 429 (2008).

[7] W. Guerin, F. Michaud, and R. Kaiser, Phys. Rev. Lett. 101, 093002 (2008). 
[8] H. Cao, Y. G. Zhao, S. T. Ho, E. W. Seelig, Q. H. Wang, and R. P. H. Chang, Phys. Rev. Lett. 82, 2278 (1999).

[9] H. Cao, J. Y. Xu, S.-H. Chang, and S. T. Ho, Phys. Rev. E 61, 1985 (2000).

[10] S. Mujumdar, M. Ricci, R. Torre, and D. S. Wiersma, Phys. Rev. Lett. 93, 053903 (2004).

[11] K. L. van der Molen, R. W. Tjerkstra, A. P. Mosk, and A. Lagendijk, Phys. Rev. Lett. 98, 143901 (2007).

[12] J. Andreasen et al., e-print arXiv:1001.4671v1 [cond-mat.dis$\mathrm{nn}]$.

[13] C. Vanneste, P. Sebbah, and H. Cao, Phys. Rev. Lett. 98, 143902 (2007).

[14] H. E. Türeci, L. Ge, S. Rotter, and A. D. Stone, Science 320, 643 (2008).

[15] H. Cao, Y. Ling, J. Y. Xu, and A. L. Burin, Phys. Rev. E 66, 025601(R) (2002).

[16] J. Fallert, R. J. B. Dietz, J. Sartor, D. Schneider, C. Klingshirn, and H. Kalt, Nat. Photonics 3, 279 (2009).

[17] S. John and G. Pang, Phys. Rev. A 54, 3642 (1996).

[18] G. van Soest, F. J. Poelwijk, R. Sprik, and A. Lagendijk, Phys. Rev. Lett. 86, 1522 (2001).

[19] H. E. Türeci, A. D. Stone, and B. Collier, Phys. Rev. A 74, 043822 (2006).

[20] R. W. Tjerkstra, J. G. Rivas, D. Vanmaekelbergh, and J. J. Kelly, Electrochem. Solid State Lett. 5, G32 (2002).
[21] M. P. van Albada and A. Lagendijk, Phys. Rev. Lett. 55, 2692 (1985).

[22] P.-E. Wolf and G. Maret, Phys. Rev. Lett. 55, 2696 (1985).

[23] F. J. P. Schuurmans, Ph.D. thesis, University of Amsterdam, 1999.

[24] E. Hecht, Optics (Addison Wesley, Reading, MA, 2002).

[25] W. H. Press, S. A. Teukolsky, W. T. Vettering, and B. P. Flannery, Numerical Recipes: The Art of Scientific Computing (Cambridge University Press, Cambridge, UK, 2007).

[26] B. Shapiro, Phys. Rev. Lett. 57, 2168 (1986).

[27] I. Freund and D. Eliyahu, Phys. Rev. A 45, 6133 (1992).

[28] S. Mujumdar, V. Türck, R. Torre, and D. S. Wiersma, Phys. Rev. A 76, 033807 (2007).

[29] X. Wu and H. Cao, Opt. Lett. 32, 3089 (2007).

[30] H. Cao, X. Jiang, Y. Ling, J. Y. Xu, and C. M. Soukoulis, Phys. Rev. B 67, 161101(R) (2003).

[31] V. S. Letokhov, Sov. Phys. JETP 26, 835 (1968).

[32] A. Yamilov, X. Wu, H. Cao, and A. L. Burin, Opt. Lett. 30, 2430 (2005).

[33] A. Siegman, Lasers (University Science Books, South Orange, NJ, 1986).

[34] S. Faez, A. Strybulevych, J. H. Page, A. Lagendijk, and B. A. van Tiggelen, Phys. Rev. Lett. 103, 155703 (2009). 